\title{
Translation and cultural analysis of the epic "Datu Somangga and Bubu of Huamianun" of Francisco I. Alzina
}

\author{
Amat, Aldwin B
}

Leyte Normal University, Philippines (aldwinamat777@gmail.com)

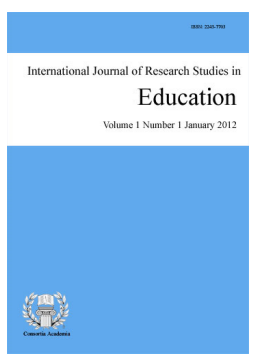

Accepted: 25 October 2020 Online ISSN: $2243-7711$

OPEN ACCESS

\section{Abstract}

This study aims to translate into Waray language the epic of Francisco I. Alzina which was written in English, titled, Datu Somangga and Bubu of Huamianum. Part of this study is also to analyze the cultural terms and values found in the translated epic. Content analysis was used as the research design by the researcher. The reactors of the study used the rubric in translation by the Department of Education. Based on the results of the study, the epic was translated into Waray language and the basic rules and principles of translation were applied. In terms of the cultural values, the high regard on women was very evident when it comes to the process of courtship by men, the bravery and determination of a man to win a woman's heart, and giving high value on a Waray woman's dignity. There are also cultural terms found in the epic which is reflected in the current lives of the Waraynon such as buyo, apog, bunga, pangaiao, agta, and balangay. However, there are cultural terms which are also considered as old terms but are still reflected in the lives of Waraynon such as pinanta, pugul, binocot, bubu, and sombol. Based on the results of the study, it is recommended to conduct fundamental steps on translating local and regional literature written in English, to be fully understood by the Waraynon and can be used in teaching MTB-MLE courses/subjects.

Keywords: epic; translation; analysis; values; cultural terms 


\section{Translation and cultural analysis of the epic "Datu Somangga and Bubu of Huamianun" of Francisco I. Alzina}

\section{Introduksyon}

Ang mga tao sa Silangang Visayas tulad sa ibang rehiyon ng Pilipinas ay may natatagong yamang kultural na nagbigay ng pagkakakilanlan bilang mga Pilipino. Isa na sa yamang ito ay ang mga akdang pampanitikang Leynete-Samarnon na isinulat ng mga katutubong manunulat ng rehiyon VIII. Magkagayunpaman, ang mga ito ay nasusulat lamang sa wikang Ingles nang dahil na rin sa kakulangan ng kamalayan at oryentasyong lokal ng mga akdang pampanitikan. Bunga nito, ay patuloy pa rin tayong nanghihiram at tumatangkilik ng mga babasahin mula sa ibang rehiyon ng Pilipinas.

Kung kaya, ipinagtibay ng Kagawaran ng Edukasyon Atas Bilang 74, Serye 2009 ang paggamit ng mother tongue sa pagtuturo batay sa Multilingual Education, na kung saan isinaad dito na ang lahat ng mga panlalawigang direktor at superintendente ay hinihikayat na palawakin at paunlarin ang pagpapatupad ng sistemang "Multilingual Education" sa ilalim ng programang "School-Based Management" sa pamamagitan ng pagsuporta ng mga lokal na pamahalaan sa pagbuo ng mga tiyak na plano para sa pangangasiwa at pagtataguyod nito. Ipinatupad din dito ang malawakang pagsasanay para sa mga guro na maging bihasa sa pagtuturo sa mga mag-aaral na nagsisimula pa lamang mag-aral sa elementaya gamit ang vernakular. Binigyang-pansin din dito ang pagpapalimbag ng mga babasahing vernakular, mga gawaing pagsasanay ng mga mag-aaral at iba pang kagamitang panturo. Mahigpit na ipinatupad ang batas na ito pagkaraan ng tatlong taon nang puspusang pagsusuri at ebalwasyon para sa malawakang pag-aaral.

Ang ibang manunulat at ang mga pantas ng karunungan ay nagsagawa rin ng pag-aaral kung paano ipakilala at ipasok sa pananaw at damdamin ng mga mag-aaral na tayo ay may pagkakakilanlan bilang mga Pilipino. Ganoon din sa pag-aaral na ito, ang tungkol sa pagpapayaman ng mga akdang lokal sa pamamagitan ng pagsasasalin, na kung saan ay isinalin sa wikang Leynete-Samarnon ang isang babasahing nasusulat sa Ingles na ang nilalaman ay tungkol sa kulturang Winaray. Mahalagang maisalain at masuri ang mga pagpapahalagang kultural na nakapaloob sa epiko upang maunawaan ito ng mga Waraynon at maipasa sa susunod na henerasyon ang karunungang yaman ng panitikang lokal.

Sa aklat ni Villafuerte et al. (2006), nabanggit ni F. Sionil mula sa kanyang pag-aaral na ang panitikang Tagalog, Cebuano at Ilokano ay patuloy na lumalago at ang mga manunulat nito ay lalong dumami dahil sa naging bihasa na sila sa paggamit ng mga wikang ito. Ilan sa mga akdang nasusulat sa ilang wika tulad ng Pampango at Pangasinan ay unti-unti na ring naglalaho.

Hindi man binanggit dito ang wikang Winaray kundi ang mga wikang Pampango at Pangasinan ay iniuudyok tayong mga Waraynon na gumawa ng sariling hakbang upang pagyamanin ang mga akdang lokal. Kaugnay nito ay ang pagsasalin sa mga akdang nasusulat sa Ingles na ang nilalaman ay kulturang Winaray upang masalamin ang ating pinagmulan at gumawa ng mga hakbang upang ipakilala at ituro ng mga guro ang mga ito sa kanilang mga mag-aaral. Malaking hamon ang talumpati ni Carlos P. Garcia sa Kumbensyon ng Samahan ng mga Guro sa Pilipinas, na ayon sa kanya ang ating mga guro ay dapat tumingin-pasulong, siyasatin ang kaluluwa ng mga Pilipino at hanapin ang pinakamabuting bunga ng ating kultura, kung saan doon ibabatay ang ituturo sa mga mag-aaral. Ang mga namanang mabubuting pag-uugali tulad ng pagkakaisa ng pamilyang Pilipino, paggalang sa batas at kautusan, kagandahang-loob gaya ng kabayanihan ay dapat ipasok sa kurikula at bumuo ng mga kagamitang panturo.

Talagang napakahirap bumuo ng mga kagamitang panturo lalo na kung ang pagsasalin sa mga akdang Leynete-Samarnon ang pag-uusapan. Subalit, ito lamang ang paraan upang ipamalas at ipaunawa sa mga bata na 
mayaman ang kulturang Pilipino tulad ng mga akdang pampanitikan na nagbubunsod sa atin upang magkaroon tayo ng pagkakakilanlan. Ganito ang nais iparating sa mga suliraning kinakaharap ng mga guro sa wika at panitikan na sa pagsasaling-wika, ang kakulangan ng kaalaman sa kultura (cultural translation) ay hindi gaanong mabigat na problema, kundi kung paano natin unawain at tanggapin ang mga naisaling akda upang maging kapaki-pakinabang at katanggap-tanggap ang kulturang ating pinagmulan (Ok, 2003). Ayon pa sa kanya, kung ang mga wikain ay nagsisilbing daan para sa pagkakakilanlan ng ating kultura ay dapat matanto ng mga mag-aaral na ang mga aklat at iba pang mga babasahin ay nagsisilbing tulay upang makapag-ambag ng kaalaman para sa pagkatuto ng mga mag-aaral sa kulturang kanilang pinagmulan.

Ito ay nagsilbing patunay lamang na tayong mga Waraynon ay dapat gumawa ng hakbang upang mapagyaman ang panitikang lokal ar rehiyonal. Kung ang mga ito ay nasusulat man sa wikang Ingles dapat lamang gumawa tayo ng paraan upang ito`y maisalin sa wikang rehiyonal at bumuo ng kagamitang panturo na sasalamin sa kabataan Pilipino na mayaman tayo sa mga akdang pampanitikan. Binanggit sa pag-aaral ni Tupaz (2001) na,

Ang pagsasalin sa wikang Filipino ng mga orihinal na nakasulat at 'di nakasulat ng mga literatura'y isa sa mga hakbang tungo sa pagpapayaman ng mga kagamitan sa pagtuturo, gayunpaman ay magiging bahagi ng kulturang- mana ng ating lahi.

Kaugnay nito, ang pagsasalin at pagsusuring kultural ng epikong Winaray na nasusulat sa Ingles ay tugon upang mapagyaman ang panitikang lokal sa pamamagitan na rin ng pagbuo ng kagamitang panturo upang magamit sa pagtuturo ng panitikang rehiyonal.

May mga batas at ilang programa ng pamahalaan na nagtataguyod sa preserbasyon at pagpapayaman ng panitikang lokal tulad na lamang Memorandum Pansangay Blg. 142, Serye 1999, na humingi ng kooperasyon sa mga kinauukulang larangan na magsumite ng talaan ng mga salitang Leynete-Samarnon na may katangiang kultural sa iba`t ibang lugar ng Leyte.

Isinaad din sa Republic Act No. 7356 nabuo ang National Commission for Culture and the Arts (NCAA) na may mandatong maitaguyod at maprotektahan ang makasaysayang kultura at pamana ng bansa. Samakatuwid, ipinaabot nito ang pagpapayaman ng panitikang lokal sa pamamagitan ng paglilikom, pagsasalin at gawaing pagsusuri bilang bahagi ng kulturang Pilipino. Sa batas na ito, matutugunan ang misyon ng Kagawaran ng Edukasyon na nakasaad sa Akta Republika 10533 o ang Pinaunlad na Pangunahing Edukasyon 2013 ang lokalisasyon at kontekstuwalisasyon ng mga aralin sa pagtuturo nakapaloob sa Sekyon 10.2:

The DepEd shall adhere to the following standards and principles, when appropriate in developing the enhanced basic education curriculum. (d) The curriculum shall have contextualized and global and $(h)$ The curriculum shall be flexible enough to enable and allow schools to localize, indigenize and enhance the same base on their respective educational and social context.

Isang panawagan ito sa mga bumabalangkas ng kurikulum kasama na ang mga guro na gumawa ng hakbang na bumuo ng kagamitang panturo mula sa kani-kanilang lugar upang matugunan ang kahingian ng kurikulum. Kaugnay nito, mahalagang mapayaman ang panitikang rehiyonal sa pamamagitan ng pagsalin at pagsusuring kultural ng epiko ni Francisco I. Alzina sapagkat wala pang naitalang epiko sa Rehiyon VIII na nasusulat sa wikang Winaray. Nakalulungkot din na karamihan ng mga guro at mag-aaral sa Leyte at Samar ay hindi nakakaalam sa epikong ito kundi ang epikong nababasa lamang sa mga lumang aklat ng Panitikan katulad ng Lam-ang, Ibalong at iba pa. Kaya minabuti sa pag-aaral na ito na isalin sa Winaray ang epikong ito upang malaman ng mga mag-aaral na may epiko ang Silangang Visayas at mabatid din nila ang mga pagpapahalagang kultural na naglalarawan sa katangian ng mga Waraynon. 


\subsection{Layunin ng pag-aaral}

Nagsilbi bilang mga tiyak na layunin sa pag-aaral ang sumusunod:

$>\quad$ Masuri ang isinagawang salin sa Winaray ng epikong "Datu Somangga and Bubu of Huamianun" ni Franciso I. Alzina.

$>\quad$ Masuri ang mga pagpapahalagang kultural at mga salitang kultural mula sa isinaling epiko.

\section{Metodolohiya}

Ang pag-aaral na ito ay ginamitan ng desinyong deskriptibong-kwalitatibo sapagkat dito inilapat ang pagsusuri sa isinagawang salin ng epikong Leyte-Samar na pinamagatang "Datong Somangga and Bubu of Huamianun" ni Francisco I. Alzina. Naging batayan sa paglikom ng akda ang sumusunod: (a) natatanging epiko ng Silangang Visayas na nasusulat sa Ingles na kakikitaan ng mayamang kultura ng Waraynon (b) mga salitang kultural na nakapaloob sa epiko at (c) pag-uugali ng mga Waraynon na makikita sa epiko. Nabibilang din sa pag-aaral na ito ng indehinus na pamamaraan sapagkat tinalakay sa pagsusuri ang mga simbolo, kaugalian, katangian at iba pang saklaw ng kulturang `di materyal. Nasa purposive ang paraan sa pagkapalap ng akda sa dahilang hindi pamilyar sa mga Waraynong mag-aaral ang epikong ito at kakikitaan din ito mayamang simbolong kultural tulad ng apog, buyo, bunga at iba pa na makikita sa pamumuhay ng mga Waraynon.

Sa gawaing pagsasalin, isinagawa ang unang pagbasa ng salin upang makuha ang pangkalahatang kaisipan, diwa at mensahe, paulit-ulit itong binasa upang lubos itong maunawaan ng tagasalin. Itinala ang mga salitang kultural, mga pahayag, idyomatiko at kauring pahayag. Isinagawa ang salin, pinanatili ang mga salitang kultural at inilarawan ang kahuluguan nito. Inirebisa ang unang burador at pagkatapos ng isang linggo ay ipinasuri ito sa dalawang eksperto sa wikang Ingles at Waray gamit ang rubric sa pagsasalin ng certificate program for non-specialized in Filipino ng DepEd ng Leyte Normal University sa loob ng isang linggo ay kinuha ito sa kanila upang maayos ang mga suhestiyon at puna sa salin. Muling inirebisa ang isinaling epiko at ipinasuri uli sa dalawang eksperto, sinunod ng tagasalin ang ilang puna at suhestiyon ng mga ekspersto at muling ipinasuri uli sa kanila. Sa pagsasapino ng salin ay muling ipinasuri ito sa mga eksperto, sa huling yugto ng pagsasalin ay nasunod ng tagasalin ang ilang puna at suhestiyon ng mga eksperto. Pagkatapos naisapino ang salin ay sinuri ang mga salitang kultural at pagpapahalagang kultural na nakapaloob sa isinaling epiko. Ibinatay sa pagsusuring kultural ang pitong pangunahing pagpapahalagang pagpapakatao ng DepEd. Values Education Program (VEP) of 1997. Sinuri ito batay sa dimensyong pisikal, espirituwal, intelektuwal, politikal, sosyal, moral, at ekonomik.

\section{Mga resulta at diskusyon ng pag-aaral}

\section{Talahanayan 1}

Unang reaksyon ng mga eksperto sa wikang Waray at Ingles

\begin{tabular}{|c|c|c|}
\hline Kraytirya & Mean & Pagpapakahulugan \\
\hline $\begin{array}{l}50 \% \text { ng kaisipan at diwa ng orihinal na akda ay naisalin } \\
\text { sa patutunguhang wika }\end{array}$ & 5.00 & lubos na sumasang-ayon \\
\hline $\begin{array}{l}\text { Nagamit ang iilang tuntuning panggramatika ng } \\
\text { pinagsasalinang wika }\end{array}$ & 3.50 & pag-aalinlangan \\
\hline $\begin{array}{l}\text { Walang gaanong kaisahan, kawastuhan, at ugnayan ang } \\
\text { bawat pangungusap at talata }\end{array}$ & 4.00 & sumasang-ayon \\
\hline Kabuoan & 4.13 & sumasang-ayon \\
\hline
\end{tabular}

Mula sa talahanayan nabatid ang naging resulta sa isinagawang unang pagbasa at pagsusuri ng salin ng dalawang eksperto sa wikang Ingles at Waray na nagsilbing reaktor sa isinaling epiko. Lumabas mula sa kanilang matiyagang pagsusuri at pamumuna na ang nilalaman at diwa ng salin ay naisalin lamang sa

4 Consortia Academia Publishing (A partner of Network of Professional Researchers and Educators) 
Translation and cultural analysis of the epic "Datu Somangga and Bubu of Huamianun” of Francisco I. Alzina

limampung bahagdan (50\%) mula sa orihinal na wika (Ingles) patungo sa target na wika (Waray) kung kaya kapwa nila itong lubos na sinang-ayunan para sa pagsasaayos ng diwa at nilalaman sa isinaling epiko. Nakita rin ng unang reaktor na nagamit lamang ang iilang tuntuning panggramatika ng pinagsasalinang wika kaya siya lubos na sumasang-ayon. Ganoon din ang pangalawang reaktor, na kung saan ay nagkaroon ng alinlangan sa lahat ng mga tuntuning panggramatika na ginamit ng tagasalin sapagkat ayon sa kanya ay wala pang standard na pag-aaral sa ortograpiyang Winaray. Lumabas din sa pagsusuri na sumang-ayon ang unang reaktor na wala pang gaanong kaisahan, kawastuhan ang ugnayan ng mga pangungusap at talata kung kaya nagbigay pa siya ng mga payo kung paano mapabuti ang salin. May alinlangan din ang pangalawang reaktor sa ugnayan ng bawat pangungusap at talata sa isinaling epiko sapagkat hindi nagkatugma sa orihinal na talata ang pagkakahanay ng mga talata sa isinagawang salin kung kaya napag-isipan ng pag-aaral na ito na isaalang-alang pa ito para sa pangalawang pagsusuri ng salin sa tulong na rin ng mga reaksyon at suhestyon ng mga reaktor.

\section{Talahanayan 2}

Ikalawang reaksyon ng mga eksperto sa wikang Waray at Ingles

\begin{tabular}{|c|c|c|}
\hline Kraytirya & Mean & Pagpapakahulugan \\
\hline $\begin{array}{l}75 \% \text { ng kaisipan at diwa ng orihinal na akda ay naisalin } \\
\text { sa patutunguhang wika }\end{array}$ & 5.00 & lubos na sumasang-ayon \\
\hline $\begin{array}{l}\text { Nagamit ang nakararaming tuntuning panggramatika ng } \\
\text { pinagsasalinang wika }\end{array}$ & 5.00 & lubos na sumasang-ayon \\
\hline $\begin{array}{l}\text { May kaisahan, kawastuhan, at ugnayan ang bawat } \\
\text { pangungusap at talata }\end{array}$ & 5.00 & lubos na sumasang-ayon \\
\hline Kabuoan & 5.00 & lubos na sumasang-ayon \\
\hline
\end{tabular}

Tala. Rubrik ng pagsasaling-wika ng certificate program for non-specialized in Filipino ng Department of Education (Leyte Normal University, 2008).

Makikita sa talahanayan ang isinagawang pangalawang suri at basa ng salin ng dalawang eksperto sa wikang Ingles at Waray. Dito isinaayos nila ang mga kahinaan at kamaliaan ng salin mula sa naunanag pagsasagawa ng pagsusuri sa parehong reaktor. Mula sa kanilang pagbabasa at pamumuna ay napansin ng dalawang reaktor na nagkaroon na ito ng pagbabago mula sa limampung porsyento (50\%) ng mga kaisipan at diwa ng orihinal na akda ay naisalin na ito sa patunguhang wika ng pitumpo at limang porsyentong pagababago (75\%) kung kaya napagkasunduan ng dalawang reaktor na lubusang sumang-ayon para sa mga nilalaman at diwa ng salin. Sa gramatika naman ay kapwa lubos na sumang-ayon ang dalawa na nagamit na ang nakararaming tuntuning panggramatika ng pinagsalinang wika. Lubos din na sumang-ayon ang dalawang reaktor na nasunod na ng tagasalin ang kanilang mga puna para sa kaisahan, kawastuhan, at ugnayan ng bawat pangungusap at talata para saa naisaling epiko. Magkaganoon paman ay hindi pa rin maiiwasang magbigay ng kaunting puna ang dalawang reaktor. Kaya napagdesisyunan na pag-aaral na ito at ganoon din ng mga manunuri na idaaan pa ito para sa pagsasapino ng salin dahil na rin sa kaunting kamalian gaya ng paglalagay ng tuldik para mabigkas nang wasto sa Waray, maging ang kaunting kamalian sa ispeling dahil sa tipograpikal na pagkakamali.

\section{Talahanayan 3}

Huling yugto sa pagbasa ng mga eksperto sa wikang Waray at Ingles

\begin{tabular}{|c|c|c|}
\hline Kraytirya & Mean & Pagpapakahulugan \\
\hline $\begin{array}{l}\text { Lahat o } 100 \% \text { ng kaisipan at diwa ng orihinal na akda ay } \\
\text { naisalin sa patutunguhang wika }\end{array}$ & 5.00 & lubos na sumasang-ayon \\
\hline $\begin{array}{l}\text { Nagamit ang lahat ng tuntuning panggramatika ng } \\
\text { pinagsalinang wika }\end{array}$ & 5.00 & lubos na sumasang-ayon \\
\hline $\begin{array}{l}\text { May ganap na kaisahan, kawastuhan, at ugnayan ng } \\
\text { bawat pangungusap at talata }\end{array}$ & 5.00 & lubos na sumasang-ayon \\
\hline
\end{tabular}

Tala. Rubrik ng pagsasaling-wika ng certificate program for non-specialized in Filipino ng Department of Education (Leyte Normal University, 2008).

Ang talahanayan na ito ay huling yugto sa isinagawang salin, sa yugtong ito dumaan ng pagsasapino at pagsasapinal ng salin. Ang mga kaunting kamalian sa isinaling epiko ay dapat maayos para sa panghuling 
Amat, A. B.

gawain ng pagsasaayos ng salin. Mula sa naisapinong salin ay nakita ng dalawang reaktor na ang lahat ng kaisipan at diwa ng orihinal na akda ay naisalin na nang mabuti sa patunguhang wika. Kaya kapwa sila lubos na sumang-ayon dito. Napansin din ng mga reaktor na ang lahat ng mga tuntuning panggramatika ay nasunod na ng mananalin kaya lubos nila itong sinang-ayunan. Ang pagkakasunod-sunod o organisasyon ng salin ay kapwa nasuri na rin ng mga reaktor kung kaya ay lubos nilang sinang-ayunan na nagkaroon na ito nang ganap na kaisahan, kawastuhan, at ugnayan ng ng mga pangungusap at talata sa naisaling epiko.

\section{Talahanayan 4}

Mga pagpapahalagang kultural sa epikong Datong Somangga at Bubu of Huamianon

\begin{tabular}{|c|c|c|}
\hline $\begin{array}{c}\text { Mga Pangyayari sa Epiko na Sumisimbulo sa } \\
\text { Kulturang Waraynon }\end{array}$ & Dimensyong Kultural & Pagpapahalagang Kultural \\
\hline $\begin{array}{l}\text { May isang binocot (prinsesa) na Huamianon (tawag sa } \\
\text { taong nakatira sa isla na matatagpuan sa Silangang } \\
\text { Visayas) may magandang reputasyon at pinakatanyag sa } \\
\text { lahat ng magaganda sa islang iyon. Nakatira siya sa isang } \\
\text { bahay na saradong-sarado kaya hindi siya malapitan } \\
\text { upang kausapin. Maihahalintulad siya sa isang araw na } \\
\text { kapag sumikat ang lahat ay makakaalam. }\end{array}$ & Dimensyong Politikal & $\begin{array}{l}\text { Mataas na pagkilala at pagpapahalaga } \\
\text { sa kapangyarihan at kakayahan ng } \\
\text { kababaihan. }\end{array}$ \\
\hline $\begin{array}{l}\text { Nagsimula ang kwento ng epiko sa panghaharana ng Datu } \\
\text { sa isang tanyag na prinsesang nakatira sa isla ng } \\
\text { Huamianon. Isang kaugalian sa panliligaw ay ang } \\
\text { humingi ng pahintulot sa panghaharana at ang } \\
\text { magpakilala sa prinsesa. Para sa mga taga-Isla, itinuturing } \\
\text { na kabastusan at insulto sa babae ang hindi pagsunod } \\
\text { ditto. }\end{array}$ & Dimensyong Sosyal & $\begin{array}{l}\text { Noon pa man ay bahagi na ng } \\
\text { kulturang Pilipino ang panghaharana } \\
\text { na makikita mismo sa epiko. Sa } \\
\text { kasalukuyan, mahigpit na } \\
\text { pinaalalahanan ng mga magulang ang } \\
\text { kanilang anak na dalaga na kung } \\
\text { liligawan sila ng lalaki ay dapat sa } \\
\text { bahay nila upang hindi siya mabastos. }\end{array}$ \\
\hline $\begin{array}{l}\text { Upang matupad ang hangarin ng Datu na mapangasawa } \\
\text { ang prinsesa ay agad niyang sinunod ang mga hamon nito, } \\
\text { katulad na lamang na manguha na mga buyô, apog, goma, } \\
\text { bunga, daang-daang bihag sa pakikidigma mula sa mga } \\
\text { isla ng Mindanao at kaharian ng Tsina, upang ihandog sa } \\
\text { dalaga at pagpunta sa ginduutan sa langit (sulok ng langit } \\
\text { at lupa) upang kunin ang mga ilaw ng kidlat. }\end{array}$ & Dimensyong Sosyal & $\begin{array}{l}\text { Ipinapakita dito na ang mga Waraynon } \\
\text { ay matapang sa mga hamon sa buhay. } \\
\text { Katulad na lamang sa mga } \\
\text { pakikidigmang pinagdaanan ng Datu. } \\
\text { Larawan ng tunay na lalaking } \\
\text { Waraynon ang pagiging matapang at } \\
\text { determinasyon na makamit ang } \\
\text { kanyang mithi para sa kanyang } \\
\text { minamahal. }\end{array}$ \\
\hline $\begin{array}{l}\text { Ang pinagkakatiwalaang agta (ita) ang naging mensahero } \\
\text { ng Datu kung ano ang mga pagsubok sa panliligaw na } \\
\text { ipinagagawa ng prinsesa para sa datu. Inilahad sa epiko na } \\
\text { ang pangaiao o pakikidigma ng Datu upang manghuli ng } \\
\text { bihag para sa prinsesa ay katulong ang kanyang } \\
\text { nasasakupang ita hanggang Gran China, ang sakayang } \\
\text { pandagat ay tinatawag na balangay, ito ay binubuo ng } \\
\text { Datu, katiwalang ita at mga mandirigma. }\end{array}$ & Dimensyong politikal & $\begin{array}{l}\text { Sa sistemang politikal, noon pa man } \\
\text { ay may herarkiyang bumubuo sa } \\
\text { lipunan: Ang datu, katiwalang agta ng } \\
\text { datu, katulong at mga mandirigma. }\end{array}$ \\
\hline $\begin{array}{l}\text { Ang sombol ay malaking balahibong inilalagay sa dulo ng } \\
\text { sakayan, palamuti itong inilalagay bilang simbolo ng } \\
\text { tagumpay na nagawa ng Datu lahat ng pagsubok ng } \\
\text { prinsesa. }\end{array}$ & Dimensyong sosyal & $\begin{array}{l}\text { Sa kulturang Pilipino, ang paghihintay } \\
\text { ng magkasintahan na makapagtapos } \\
\text { ng pag-aaral bago mag-asawa ay } \\
\text { itinuturing nilang tagumpay na lahat } \\
\text { ng mga pagsubok sa buhay ay } \\
\text { kanilang nalampasan. Nakita sa istorya } \\
\text { na ang mga palamuti tulad ng sombol } \\
\text { ay sumisimbilo sa diploma, degree } \\
\text { muna bago asawa. }\end{array}$ \\
\hline $\begin{array}{l}\text { Nang magawa ng Datu lahat ng ipinag-utos ng prinsesa na } \\
\text { manguha ng buyô, mga taong bihag, maliban na lamang } \\
\text { sa mga ilaw ng kidlat sa sulok ng langit ay nabahala siya } \\
\text { baka sugurin siya ng Datu at alisin sa kanyang ulo ang } \\
\text { bangoloy o pinanta (hindi totoong buhok na isinusuot } \\
\text { noon ng kababaihan upang madagdagan ang kanilang } \\
\text { ganda). Malaking insulto para sa prinsesa kapag } \\
\text { matanggal ito ng kanilang katunggali, isang kabastusan at } \\
\text { malaking kahihiyan ito sa kanya. }\end{array}$ & $\begin{array}{l}\text { Dimensyong pisikal } \\
\text { Dimensyong Politikal }\end{array}$ & $\begin{array}{l}\text { Pananalig sa kagandahang pisikal. } \\
\text { Pangangalaga sa reputasyon ng } \\
\text { pagkababae. }\end{array}$ \\
\hline $\begin{array}{l}\text { Nang malaman ng prinsesa na natupad ng Datu lahat ng } \\
\text { kanyang hamon ay umiyak ito at inihanda niya ginto } \\
\text { bilang pugol o tanda ng kanyang pakikipag-isang dibdib } \\
\text { sa asawa at sila ay namuhay nang maligaya at marangya } \\
\text { dahil sa kanilang kayamanan. }\end{array}$ & Dimensyong Sosyal & $\begin{array}{l}\text { Ang pugol o tanda ng kasal sa epiko } \\
\text { ay inilalarawan sa kontekstog Pilipino } \\
\text { ang dore o mga regalong inihahandog } \\
\text { ng lalaki sa babae. Sa epiko, ang dore } \\
\text { ng lalaki ay daang-daang bihag na } \\
\text { inihandog niya sa prinsesa. Bilang } \\
\text { kapalit sa kanyang sakripisyo ay } \\
\text { inihanda ng prinsesa ang mga ginto } \\
\text { para sa kanilang pag-iisang dibdib. }\end{array}$ \\
\hline
\end{tabular}

6 Consortia Academia Publishing (A partner of Network of Professional Researchers and Educators) 


May ilang lalawigan sa Pilipinas na
kaugalian pa rin ang dore na hinihingi
ng mga magulang ng babae sa lalaki.
Kung gaano kahalaga at kamahal ang
kanilang anak na babae ay ganoon din
ang halagang katumbas ng dore. Sa
kasalukuyan, mas pinipili ng mga
magulang ng babae na may
pinag-aralan ang lalaki upang
mabigyan ng kinabukasan ang babae.

Mula sa isinaling epiko ay mababatid ang mayaman na pagpapahalagang kultural sa panliligaw ng Datu sa bubu o binocot (prinsesa). Nabibilang sa dimensyong sosyal ang ilan sa mga kaugalian na naging bahagi na sa kulturang Pilipino tulad sa paghingi ng pahintulot sa dalaga bago umakyat-ligaw ang binata, inilarawan sa epiko ang tamang asal sa pagpapakilala ng Datu sa dalaga bago siya mangharana. Nabibilang din sa konseptong Pilipino na ang panliligaw o panunuyo sa dalagang Pilipina ay dapat dumaan muna sa mga pagsubok ng dalaga bago masabing dalisay ang pagmamahal nito ng binata, mababatid sa epiko ang mahihirap na hamon ng prinsesa sa Datu tulad ng pananalakay ng mga bihag sa mga isla ng Mindanao, China at pagpunta sa kalangitan upang kunin ang mga ilaw ng kidlat.

Samantala nabibilang sa dimensyong politikal ang mataas na pagkilala at pagpapahalaga sa kapangyarihan at kakayahan ng kababaihan. Mababatid sa epiko na ang prinsesa ay parang binocot o inilayo sa karamihan na kung saan walang makakakita sa kanya maliban na lamang sa kanyang mga katiwala at katulong, inihambing ito sa araw na kapag sumikat ito, ang lahat ay makakakita sa kanya. Bukod sa kanyang pagiging tanyag at maganda, may angking talino din ang prinsesa kaya hinahangaan siya ng nakararami. May mga pangyayari rin sa epiko na nagpapakita ng herarkiyang pamamahala, tulad na lamang sa mga katiwala at katulong ng datu sa panliligaw sa prinsesa. Sa pangaiao o pananalakay sa mga kalaban upang gawing bihag ng Datu upang ihandog sa prinsesa, lulan ang kanilang balangay (sakayang pandagat) sa paglalayag, ang barangay ay pinamumunuan na ng Datu, mga katiwala at katulong na agta. Nauugnay rin sa dimensyong ito ang pagsuot sa ulo ng pinanta o tagabohok ('di totoong buhok), ang nagsusuot lamang nito ay ang taong nabibilang sa mataas na estado ng lipunan tulad na lamang sa prinsesa, mababatid sa epiko ang pagkatalo ng prinsesa sapagkat natupad lahat ng pagsubok na ipinagagawa niya sa Datu. Sa kanyang pagkabahala na baka alisin sa kanyang ulo ang pinanta lalo na`t napakalaking kahihiyan at insulto ito para sa kanya ay inihanda niya ang ginto at ibinigay niya ito sa Datu bilang tanda ng kanyang pakikipag-isang dibdib. Ang pagsuot din ng pinanta ay nabibilang din sa dimensyong pisikal sapagkat makikita rito ang pananalig sa kagandahang pisikal ng prinsesa, ayon sa paglalarawan ni Alzina ang pinanta ay `di totoong buhok na nakapagdarag ganda sa babae.

Nabibilang naman sa dimensyong ekonomiko, ang mataas na valyu ng babaeng nabibilang sa mataas na estado sa lipunan kung ito ay pangangasawahin ng Datu. Makikita sa epiko na para mabihag ang puso ng prinsesa ay kinakailangan manguha ang Datu ng maraming buyo, apog, bunga, goma, mga bihag na kaaway mula sa iba-ibang isla ng Mindanao at Gran China, maging ang mga ilaw ng kulog at gawing handog para sa babae.

\section{Talahanayan 5}

Mga salitang kultural na nakita sa epiko

\begin{tabular}{ll}
\hline \multicolumn{1}{c}{ Mga Salitang Kultural } & \multicolumn{1}{c}{ Kahulugan } \\
\hline Agta & Ang tawag sa taong pandak, maitim at kulot ang buhok, pinaniniwalaang sila ang unang taong nanirahan \\
& sa Pilipinas. \\
Apog & Dinikdik na sinunog na talukap ng susô, isa itong sangkap sa pagnganga. Sa epiko, ang mga apog, buyô, \\
& Ang tawag sa sakayang pandagat na ginagamit sa paglalayag ng mga Datu. \\
Balangay & Hindi totoong buhok, isinusuot ito noon ng kababaihan para madagdagan ang kanilang ganda. Malaking \\
Bangoloy o Pinanta & insulto ito sa prinsesa kapag matanggal ito sa kanilang ulo. \\
Binocot & Ang unang tawag sa prinsesa, inilalayo ito sa tao upang hindi ito makita. \\
Bonga & Lubi-lubi ang bunga nito ay maliit pa sa niyog, isinasangkap sa pagnganganga \\
Bubu & Ang tawag sa asawa ng Datu, tinatawag din itong principal. Mula ito sa salitang Cebuano. Sa Leyte, ang \\
& unang tawag nito ay Buro o Ovu.Sa epikong ito ang salitang "bubu" ay hindi ginamit, nangangahulugan \\
& ito na "prinsesa"ang angkop gamitin.
\end{tabular}


Amat, A. B.

Talahanayan 5 ...pagtutuloy

\begin{tabular}{ll}
\hline \multicolumn{1}{c}{ Mga Salitang Kultural } & \multicolumn{1}{c}{ Kahulugan } \\
\hline Buyô & $\begin{array}{l}\text { Pinagsama-samang bunga at apog na binalot ng dahon ng buyô. Sa ibang lalawigan ang tawag nito ay } \\
\text { dalit o tilad. Kung wala ang buyông luba ang pamalit nito. } \\
\text { Tatlong klaseng bunga ito, ang apog na binalot ng dahon at ang dapon na sangkap sa pangnganga. Noon, } \\
\text { goma ang tawag nito ng mga Leyteño at Samareño sa Tagalog ay sapa minsan } u p a \text { ang tawag nito ng }\end{array}$ \\
& mga Bisaya. \\
Huamianun & Ang taong nakatira sa Huamianun na makikita sa Silangang Visayas. \\
Joangas & Mula sa salitang Intsek na chun o junco na nangangahulugan maliit na sakayang pandagat na siyang \\
& ginagamit sa paglalayag sa buong Asya. \\
Pangaiao & Palang salita sa Waray ay gumubat o makig-away. Sa Tagalog ay makipag-away. \\
Sombol & Ang tawag sa malaking balahibo ng ibon na inilalagay sa unahan ng sakayang-pandagat, nakaarko ang \\
& porma nito, nakatutulong ito upang pabilisin ang paglayag ng sakayan.
\end{tabular}

May mga salitang kultural na nakita sa epiko na nakaugat na sa kaugalian ng mga Waraynon tulad na lamang sa pagnganganga. Nabanggit sa epiko ang buyô, bunga, apog at goma, inilarawan ito bilang regalo ng datu sa prinsesa kapalit ang kanyang pakikipag-isang dibdib sa binocot o prinsesa. Sa Silangang Visayas, sa mga liblib na lugar, ang pagnganganga ay nakagawian na ng mga matatanda, pinaniniwalaan na ang pangunguya nito ay nakatutulong daw sa pagpapatibay ng ngipin. Nabanggit din sa epiko ang salitang bangoloy o pinanta o hindi totoong buhok na isinusuot ng prinsesa upang siya y lalong gumanda. Sa kulturang Waraynon, ang salitang bangoloy ay katumbas sa salitang Ingles na wig, isinusuot ito sa ulo ng ilang Pilipino bilang palamuti o pandagdag-ganda lalo na sa mga taong hindi biniyayaan ng makapal na buhok.

May mga salitang kultural din na kumakatawan sa karaniwang pamumuhay ng mga Waraynon, katulad na lamang sa sakayang ginagamit sa pangingisda, inilalarawan sa epiko ang balangay o Joangas na ginagamit sa paglalayag ng Datu para mangaiao. Sa sistemang politikal ang balangay ay tumutukoy sa kumpol ng manlalayag na binubuo ng datu, katulong, katiwala at mandirigma. Kaugnay nito, ang salitang baranggay ay nag-ugat sa salitang balanggay, sa ngayon ay binubuo ito ng kapitan, mga lupon at mga residente ng baranggay.

Binanggit din sa epiko, ang katutubong agta o ita bilang katiwala, katulong at mandirigma ng Datu. Batay sa ilang talâ sa kasaysayan ng Pilipinas, ang agta ay itinuturing bilang indigenous people o mga unang tao na nakatira sa mga isla ng Pilipinas. Nakatatak na rin sa mga Waraynon ang pagiging matapang, napatunayan ito sa epiko tungkol sa kabayanihan at tagumpay na natamo ng Datu sa pangaiao o pakikidigma at panghuhuli ng mga kalaban para gawing bihag. Sa konteksto ng mga Waraynon, ang mga taga-Silangang Bisayas ay tunay na matapang hindi sa pakikidigma kundi sa mga pagsubok at hamon sa buhay.

Samantala, may mga salitang nagbago na ang anyo dahil sa hindi na ito ginagamit ng karamihan ngunit nananatili pa rin sa konteksto ng mga Waraynon katulad na lamang ng salitang pugul na katumbas na sa kasalukuyan na dore, bangoloy na katumbas sa wig, binocot na katumbas sa prinsesa.

\section{Isinaling epiko}

\section{Datong Somangga and Bubu nga Huamianun}

There was, says the singer, a Principala of a fine name and reputation on that island named Bubu nga Huamianun. She had the greatest reputation from among all those who were beautiful and who was also renowned for her intelligence among the noble ladies. She was so closely sheltered and enclosed in her privacy that no one ever saw her unless by remote chance. Her appearance was like the sun when it casts its first rays over the world and like a flash of light ; the latter causing awe and respect, while the former, desire and happiness. A powerful Principal named Datong Somangga who was desirous of marrying her - the poem does not state whether he was of the same island or of another - arrived in person one day beneath her house and, making a song, called for the Principala without, however, going up. Among these people it is a sign of extreme rudeness to ascend without an invitation, and especially for such persons. He called her by her name and her

\section{Hi Datu Somangga ngan an Prinsesa nga Huamianun}

May-ada hadto, siring han magkaranta, usa nga prinsesa nga may pagkaharangdon an iya ngaran, nag-uukoy hiya ha usa nga isla nga an ngaran "Huamian," (Huamianun an tawag han tuminungnong hito nga isla sugad hit taga-Palo, it ngaran Palon-on, ngan it taga-Tacloban-Taclobanon). Kilala hiya kaupay ha mga maghusay ug han mga makinaadmanon hiton dida han mga labi ka harangdon nga mga kababayin-an. Duro hiya hin katinago han iya pag-ukoy nga waray gud nakakakita ha iya kundi ha panalagsa la nga higayon. An iya hulagway daw sugad han adlaw nga nasidlit ngan nahatag hit iya siyahan nga paglamrag ha kalibutan. Nakakatingala, nakakalukmay ug nakakalipay an iya kaanyag. Usa naman nga gamhanan nga datu, nga an ngaran hi Somangga, naghihingyap mangasawa ha iya (Waray igin-asoy hini nga susumaton kun ini nga datu taga dida hito nga isla o kun tagalain la nga dapit). Inmabut hiya tikang ha ganghaan ha panimalay han prinsesa ngan 
Translation and cultural analysis of the epic "Datu Somangga and Bubu of Huamianun" of Francisco I. Alzina

other names given her because She of her beauty. She was disturbed by the voice and either truly angered or feigning anger for the lack of courtesy, she ordered a servant girl to inquire who he was- perhaps it was evening time. When she learned his name, she displayed greater irritation by asking why there might not be the customary courtesy; that is, why did he come in person and did he lack Agta to command and slaves to send. Per chance did he not have someone whom he could esteem as a son, and whom he might trust as true friend in order to carry / his wish or message/?
Immediately, without replying nor saying another word, the Datu was forced to return / from where he came/ - dismissed abruptly for the time being. He then picked out a black slave - here these are esteemed very highly and are of great value as close confidants and in whom he had great trust. He urged him to be his go- between and thereby requested the buyos. From the Principala, and not to return without them.

We have already described what buyos are and how they are offered in bonu et in malo, or as a sign of consent or love, whether good or evil. And so, the Agta, as the third party, carried his master, repeating the words of courtesy and praise, which customarily and extremely polite. She replied with She replied with the same courtesy stating that she did not have either the bonga to put on the buyos or the leaf with which to wrap them. The bongas which she used could be found there where the sun rose: the leaves in which she wrapped them could be found there where the sun sets. She did not give another word.

When the reply was received by the Datu, he immediately ordered his slaves to embark: some should go and search for the bongas toward the East; others should go and search for the leaves toward the West, just as the Principala had requested. The slaves, together with the one who carried the message, carried out the mission promptly. He, therefore, handed them to her and asked that she make the buyos for his lord. In response to this, the lady answered that prepare them because she lacks the third ingredient- lime, which is necessary for the buyos and that she has it available in a certain far-off and isolated island. Receiving this answer only, he departed.

The Datu then immediately ordered that they launch vessels and sail quickly to the spot indicated in search of the said lime. The slaves, carrying out their mission, returned with all haste and brought the lime. The gloom-ridden mediator took this promptly and speedily handed it over to the lady in behalf of his master, again requesting the buyos. The answer was that she is unable to make them until his master goes in person to Tandag, a town on the coast of Caraga and there make a mangaiao and that he hand over to her all whom he may capture there. Quickly he armed his juangas and barangays with all his fighting men and embarked for Caraga, a distance of about thirty-five or forty leagues to the pueblo of Tandag. He completed his raid and captured some 120 people. Even prior to returning to his house, he sent these captives to her with the same messenger and necessary guards so that they may hand them over to his Binocot. The mediator executed this task quickly and, in return, begged for the buyos for his master, who was returning greatly tired- out from the raid. nagharana, gintawag niya an ngaran han prinsesa, kundi waray hiya umuswag. Kay didto ha ira dapit diri batasan an pagdayón kun diri ginsisiring anay, labi na hit sugad han iya kalugaringon nga pagkatawo. Nadisturbo hiya han boses han tawo nga nakadto ha gawas upod an pagkasina o pagkaaburido tungod hin kakulang hin pagtahod ha iya, ginsugo niya an iya kabulig nga babayi para panginanuon kun hin-o nga tawo an nakakaarimutaw ha gawas hadi nga katutnga han kagab-ihon. Han paghibaro la niya han ngaran hini, nagpakita lugod hiya hin duro nga kauritón pinaagi hin pagpakiana kun kay ano hin diri niya pagsunod han mga maupay nga panggawi para ha iya ngan kun kay ano nga waray man hiya magmalahimô hin pagdaup ug pagtagád ha iya? Tungod ba kay nagkukulang hiya hin agta (maitom nga tawo nga kabulig) ngan mga uripon nga iya puydi matugunan? O bangin la waray niya anak nga lalaki nga iya matatapuran sugad hin tinuod nga sangkay basi magdara han iya hingyap o mensahe.

Ha waray damo nga pulong ug istorya, napugós an datu pagbalik ngadto han iya gintikangan. Nagpilî hiya dayon hin usa nga agta nga kun diin ginkikilala ini han ngatanan han iya mga labaw nga tinapuran. Iya ini gintugon pagtuláy para ha iya nga mangaro hin buyô sugad han iya ginpapanuyo nga diri gud bumalik kun diri makadara hini.

Mahidadabihan naton an gamit han mga buyô ngan kun ginpapaunan-o ini paghalad ha kaupayan o karat-an, o diri ngani usa nga tigaman hin pagtangdo o paghigugma. Sanglit, an agtâ nagpakadto ngan naghangyo han buyô sugad han tugon han iya agaron, puno ini hin mga pamulong pagtahud ug mga pagdayaw. Nagbaton liwat an prinsesa hin sugad gihap nga pamatasan nga waray nira bonga nga ibubukag han buyô ug igpuputos man. Kay an bonga matatad-an la didto kun hain an adlaw napunias (Sinirangan), ug an mga dahon naman, adto la mahi-a-agi-an kun hain an adlaw natutunod (Katundan). Kataliwan la hito in waray na hiya may ginbuhian nga pulong.

Han nahidangat na ini nga baton ngadto han datu, dayon la nagmando hiya han iya mga uripon, pagbiling han mga bonga didto ha Sinirangan, ngan an iba nasingadto pamiling ha Katundan sugad han hangyo han prinsesa. Natuman ini dayon nga sugo han iya mga uripon, upod na an tinapuran pagdara han iya tugon. Sanglit, iginduhol han tinapuran an mga buyô ngadto han prinsesa, basi himuon an buyô para ha iya agaron. Lugod, ha pagbaton han prinsesa nagsiring hiya nga diri hiya makakahimo hito kay kulang pa han ikatulo nga kinahanglanon nga amo an apog, nga a-adto hin usa nga harayo kaupay ngan nag-uusaan la nga isla. Han pakabati gudla han baton, an sinugo lumakat.

An datu nagmando dayon nga sumakay hira han ira mga sakayan ngan kadagmitan maglayag tikadto ha lugar nga katatad-an han mga apog. An mga uripon, sugad ha pagtuman han ira mga sugo, ini dalidali bumalik dayon dara an apog. An baga masulub-on nga tinapuran nagdara dayon han apog ngadto han prinsesa ha ngaran na gihapon han iya agaron, ha utro nga higayon nangangaro na liwat ini hin mga buyô. Binmaton an prinsesa nga diri hiya makakahimo han mga buyô kun diri an iya agaron mismo an makadto ha Tandag, usa ini nga bungto ha may dapit han baybayon han Caraga ug didto mangaio (makig-agway) ngan ighatag dayon ha iya an ngatanan nga iya didto mabibihag. Iya dayon dagmit nga gin-armasan an iya mga juangas (sarakyan-pandagat) ngan an iya gihapon bug-os nga ginsasakupan (barangays) upod na liwat an iya mga mambirihag ug napakadto hira ha Caraga, nga may kahirayuon nga traynta- isingko (35) o kuwarenta (40) ka liga kun baga ha milya, syentu-singko (105) ngadto ha syentu-baynte (120) ka milya ngadto hit bungto han Tandag. Waray hira mapakyas han ira panbihag, nakabihag hira dayon hin maabot usa kagatos ngan baynte kaihap (120) nga mga tawo. San-o hiya bumalik ngadto han iya puy-anan, iginpadara lugod anay niya an mga bihag ngadto han prinsesa pinaagi la gihapon han tinapuran nga iya uripon ngan sugad man han iya mga bantay basi igdul-ong ngadto ha iya binocot (kadaan nga tawag han prinsesa nga tago ha kadam-an). Dayon ini natuman upod an paghangyo han mga buyô para ha iya agaron nga kapuy-kapuyan na tikang pala han mga pakig-away. 
Amat, A. B.

However, she was not contented with all this and urged him to go and say that she could not yet prepare the buyos until he could do the same as he had done in Tandag on the Islands of Yambig and Camiguin, which are located near the coast of Mindanao and on the western side; for the coast of Caraga lies to the east and is situated on an elevated portion of that island. The Principal without any delay, left to carry out the task, but first he strengthened his forces. After several days, he returned with his vessels filled with captives; some 235 persons of all classes were immediately sent to her through the same slave, who again pleaded for the buyos. Again, to this she reacted with obstinacy and added that he had to make the same expedition among the people of the Island of Siquijor - which the Spaniards today call Isla de Fuegos, since this is what they saw there when it was discovered - and against another Pueblo of Dapitan, which is in view from this island and is situated on an elevation of the Island of Mindanao. Hurriedly, he executed this task again and sent on to her all the captives who were not less in number than on previous occasions, although not sufficient to win her consent. In order to receive the buyos which the courtier requested of her, she sent the messenger to tell him that he had to make the same efforts i.e. raids against the Island of Jolo, which falls under another powerful ruler and whose subjects are considered as the most courageous in the entire Archipelago, as we shall relate in another place. This new challenge did not daunt his spirits, since one who loves, unless he is insane, fears as little as those who are insane. He then readied himself for the fourth attack and departed with his armada. He sailed to Mindanao and Jolo where he fought bravely and brought back many more captives than on any previous occasion. Sending all of them to her, he continued begging for the buyos. The presentation/ of these captives/ to her implied an affirmative response to the request and perhaps marriage was certain. Even this time she did not wish to prepare the buyos for him, but sent him another message through the dismayed intermediary who spoke to him in the following manner.
Kundi, waray an prinsesa makontento hito ngatanan, salit gintugon niya an tinapuran nga diri pa niya ma-i-a-andam an mga buyô samtang diri pa niya nahihimo an sugad han iya ginbuhat ha Tandag ngadto liwat ha mga isla han Yambig ug Camiguin, nga nahihimutang harani hit baybayon hit Mindanao ha may dapit Katundan, kay an baybayon han Caraga nahimumutang ha Sinirangan ngan aada hin hitaasay nga lugar hito nga isla. Waray na magpainu - ino ngan magkarag hin panahon an datu, mas ginpaburubaskog ngan ginpakurukusog pa lugod niya an iya mga puwersa ugsa lumakat pagtuman han sugo ha iya han prinsesa. Paghagos la hin pira ka adlaw han iya panmihag didto hito nga dapit, nakabalik dayon hiya nga puno an iya sakayan nga maabot hin dosyentos traynta-singko (235) kaihap nga mga magkadurudilain nga mga katawhan. Iya dayon Ini dagmit na iginpadara ngadto han prinsesa pinaagi han amo la la gihapon nga uripon nga iya sinugo, ngan utro naghangyo naman ini han mga buyô. Ha utro nga higayon, nagmatig-a la gihapon an prinsesa, ngan nagbaton hin may kaisog, upod an pagsiring nga kinahanglan pa niya buhaton an amo la gihapon na pambihag ngadto han mga taga-Siquijor - ginngangaranan han mga Espanyol nga "Isla del Fuego" kay amo ini an ira nakit-an dida han kadiskubrehi nira hito, ug sugad man liwat an bungto han Dapitan, ngan matatan-awan tikang hito nga isla nga nahihimutang dida ha may kahitas-an ha isla han Mindanao. la gihapon nga uripon nga iya sinugo, ngan utro naghangyo naman ini han mga buyô. Ha utro nga higayon, nagmatig-a la gihapon an prinsesa, ngan nagbaton hin may kaisog, upod an pagsiring nga kinahanglan pa niya buhaton an amo la gihapon na pambihag ngadto han mga taga-Siquijor - ginngaranan han mga Espanyol nga "Isla del Fuego" kay amo ini an ira nakit-an dida han kadiskubrehi nira hito, ug sugad man liwat an bungto han Dapitan, ngan matatan-awan tikang hito nga isla nga nahihimutang dida ha may kahitas-an ha isla han Mindanao. Ha kadagmitan, ginbuhat ini niya nga utro nga sugo, ngan iginpadara niya an ngatanan nga bihag nga diri maubos an kadamo han mga nahauna nga mga bihag agud la makuha an kanan prinsesa sadang nga pagtangdo. Basi makarawat an mga buyô nga ginhahangyo ha iya han tinapuran nga uripon han datu, ginpakadto niya an tinapuran pagsumat han agaron nga kinahanglan buhaton la gihapon niya an mga pambihag kontra buyô nga ginhahangyo ha iya han tinapuran nga uripon han datu, ginpakadto niya an tinapuran pagsumat han agaron nga kinahanglan buhaton la gihapon niya an mga pambihag kontra buyô nga ginhahangyo ha iya han tinapuran nga uripon han datu, ginpakadto niya an tinapuran pagsumat han agaron nga kinahanglan buhaton la gihapon niya an mga pambihag kontra naman liwat ha isla han Jolo, nga aada ha ilarom hin gamhanan nga namumuno, kanay mga sakop amo an pinakamaisog ha bug-os nga kapurupud-an, bug-os nga kapurupud-an, nga aton ig-aasoy, nga kun diin, may-ada liwat ini hindadabihan hin nahilalain nga istorya. Waray hiya kahadlok hini nga bag-o nga ayat sugad ha iya nga nahigugugma, kay iton nahigugugma labot la kun hiya may deperensya ha ulo, diri gud nahibabaro hin kahadlok bisan la gutiay sugad hiton lurong. Utro, igintaya naman niya an iya kinabuhi ha ikaupat na pagsulong ug linmakat hiya upod an iya armada. Nakadto hiya ha Mindanao ug Jolo, diin maisog hira nga nakig-away ngan nakadara dayon hira hin duru-damo pa nga mga bihag kontra han mga nahauna nga panbihag. Han pagpadara niya han mga bihag ngadto han prinsesa, padayon nga iya ginhihinangyuon an mga buyô. An pagpadará niya hin sugad kadamô. nga mga bihag in may-ada madig-on nga karuyagsidngon nga manggad naman la makarawat niya (datu) an iya pagtangdo para han pagpakasal. Bisan dida hito nga bahin, waray la gihapon hiya maghingyap paghatag hin mga buyô para han datu, lugod nagpadará na liwat hiya hin mensahe han tinapuran nga baga nahuhulop naman ngani ngan nagyakan ngada han iya agaron hin pagkasiring.

Harangdon, an prinsesa nagsiring, nga nagdadayaw hiya han imo iginpakita nga gugma ha iya, ngan naruruyagan niya an imo pagkamaisog, kundi kinahanglan mo pa mapamatud-an nga ikaw in may-ada hul-os nga paghigugma ha iya, ngan basi mas maruyagan pa niya an imo hingpit nga kaisog, may-ada niya hinbatian nga diri hirayo hini nga isla amo an bantugan nga Ginhadian han Tsina. May-ada didto mga bahandianun nga mga tawo nga kun diin nagyayakan hira hin mga magngidlis nga tingog ug an ira pamulong baga hin nagkakanta nga waray nakakaintindi," ug waray na hiya iba nga gin-asoy pa. 
Translation and cultural analysis of the epic "Datu Somangga and Bubu of Huamianun" of Francisco I. Alzina

When that lover heard this, he fitted his ships with larger ringing, added vessels, people and arms and set- out for the fifth journey to Gran China. He appeared on those coasts without any difficulty and made assaults on the less prepared towns and captured sufficient number of people to fill the ships. He returned to his own land with remarkable speed and with captives and spoils to over-lay. Quickly he sent all these to his lady with repeated pleas for the buyos. if he would perform another task first. Namely, that as he bring her from heaven something as remarkable he had already brought her from earth. He replied: "Seeing that she requests the impossible, well then, let's go to work! We shall conquer heaven itself!" If he had lived in these present opportune times for their souls he would have known the way in which he might do it more readily than simply in the conquests he had performed on earth. The Bisayans felt that there was no more of a heaven than that which is evident to the eye; nor more of the earth than the hemisphere itself. As a result, they called the latter ginduutan sa langit, which means "where the heaven is joined to the earth. He then ordered: "Let the vessels be prepared and we shall go and make an invasion of the heaven! We shall disconnect a portion of it and we shall unfold a part of one of its eight folds or copes." From this poem it can be seen that the Bisayans counted eight heavens, as did many of the neighboring people. "We shall take away one of the largest thunderbolts; we shall make away at the same time with a piece of its brilliance. And in case there is nothing else, we shall not fail to take a ray like those which are broken off when it flashes. Therefore, let us go on, let us move!' And so he embarked, but all in vain pursuit of the ever- fleeing horizons without finding anything. Nor was he able to explore it all and therefore returned in despair
He was however, assured that he had accomplished what she had commanded him; that he was able to put at her service only the thunder and lightning, although he was unable to give them to her. Also, that the many regions that he had traversed, he heard much but found very little. He added that unless she prepared the buyos for him-something that cost him so much and tired him out so greatly he would go personally and pilfer her hair. We have already
Han pakabatí hini han nahigugugma nga datu, iya dayon ginbutangan an iya sakayan hin durudagko nga mga layag, nagdugang pa hin mga sakayan- pandagat, sugad man gihapon an kadamo han iya mga tawo ug pati na an kadamo han iya mga hinganiban para han ikalima nga pagbiyahe ngadto ha Gran Tsina. Hin-agian niya an mga kabaybayunan hin waray manla damu nga pagkuri, ginsulong niya an mga magluya nga mga bungto ug nakabihag hin igula an kadamo nga mga bihag para makasadang pagpunó han sulod han ira sakayan. Bumalik hiya dayon hin kadagmitan nga dara an mga bihag ngan an iba pa nga mga hiagi. Ginpadara dayon niya ito ngatanan ngadto han iya hinigugma, upod an panginyupo nga ihatag na an mga buyô nakabihag hin igula an kadamo nga mga bihag para makasadang pagpunó han sulod han ira sakayan. Bumalik hiya dayon hin kadagmitan nga dara an mga bihag ngan an iba pa nga mga hiagi. Ginpadara dayon niya ito ngatanan ngadto han iya hinigugma, upod an panginyupo nga ihatag na an mga buyô. Kundi, an iya hinigugma waray man la mahingaruyag hini ngatanan nga iya mga nahimo, ngan lugod nagpahimo pa hin mga magkuri nga mga buruhaton kabalyo han iya mga panaad. Nagpadayon pa hiya pagpinangaro hin mga butang nga urusahon o imposible. An iya ibinaton la nga ha dayuday ngan waray duda in a-andamon niya an mga buyô kun iya buhaton anay an usa nga butang nga dad-on ngada ha iya tikang ha langit an mga butang nga magpakaruruyag sugad han iya pakadara han mga tunan-on nga butang. Binmaton an datu, "Nakikita man naton nga iton iya pinangangaro an mga urusahon nga butang- sanglit pagtrabaho kita! Aton pagsusupilon bisan pa ngani an langit!" Kun nag-ukoy hiya dinhi yana nga panahon para hit mga kalag,

hisasabtan unta niya an paagi damu nga pagkuri, ginsulong niya an mga magluya nga bungto ug nakabihag hin igula an kadamo nga mga bihag para makasadang pagpunó han sulod han ira sakayan. Bumalik hiya dayon hin kadagmitan nga dara an mga bihag ngan an iba pa nga mga hiagi. Ginpadara dayon niya ito ngatanan ngadto han iya hinigugma, upod an panginyupo nga ihatag na an mga buyô. Kundi, an iya hinigugma waray man la mahingaruyag hini ngatanan nga iya mga nahimo, ngan lugod nagpahimo pa hin mga magkuri nga mga buruhaton kabalyo han iya mga panaad. Nagpadayon pa hiya pagpinangaro hin mga butang nga urusahon o imposible. An iya ibinaton la nga ha dayuday ngan waray duda in a-andamon niya an mga buyô kun iya buhaton anay an usa nga butang nga dad-on ngada ha iya tikang ha langit an mga butang nga magpakaruruyag sugad han iya pakadara han mga tunan-on nga butang. Binmaton an datu, "Nakikita man naton nga iton iya pinangangaro an mga urusahon nga butang- sanglit pagtrabaho kita! Aton pagsusupilon bisan pa ngani an langit!’ Kun nag-ukoy hiya dinhi yana nga panahon para hit mga kalag, hisasabtan unta niya an paagi diin makakahimo pa hiya hin mas madali kontra han mga kadaugan nga nabuhat niya ha tuna. Napausa an mga Bisaya, kay bisan usa ha ira in waray pa may nahingadto ngan nakakita hin tinuod nga langit ngan bisan pa man an katubtuban han mga dampog in waray pa nira hikit-i. Sanglit, ira gintawag an sidsid hit langit nga "ginduutan han langit" nga an karuyag-sidngon, an lugar diin nagsusumpay an langit ngan han tuna. Nagmando an datu,

"Andama an mga sarakyan, makadto kita ngan aton pagpapaklion an usa hito nga bahin ha ikawalo hit iya pilo.” Dinhi hini nga susumaton, masasabtan nga an mga Bisaya, in nag-iihap hin wawalo kaihap han langit, sugad man gihapon hiton binubuhat han pag-ihap hiton binubuhat han pag-ihap hiton iba nga kahigranian han mga katawhan. Dugang pa han iya mando, sugad han pagkasiring, "Aton kukuhaon iton usa hiton pinakadako nga lilinti ug an kikilat ngan an nagkapusakpusak nga silhag hini. Ngan kun pananglit waray na gud iba, diri kita mapapakyas bisan naman la an pagkuha hin usa nga silhag sugad hadton mga nagkatipak-tipak kun nakiblat an kikilat Sanglit, tana kalakat na kita!' Ngan nagpadayon hiya paglakaton pero ha luyo han ira mga panalingkamot, napakyas hira tikang han ira paglinakat-lakat ha mga kasidsiran han langit, sugad man nga waray na gihapon nira masudoy an ngatanan, sanglit bumalik hira nga nawad-an hin paglaum.

Kundi, malipay hiya nga iya natuman an mga iginsugo ha iya labot la han dalugdog ug kikilát, nga waray niya maihatag ngadto han prinsesa. Ngan nahiabot man gihapon hiya ngadto han kadam-an ngan magkadurudilain nga dapit han kapurupud-an. Nagsiring pa lugod hiya nga labot la kun andamon niya an mga buyô, nga amo an nakapagpagusto hin duro kaupay ha iya ngan nakapagpakapoy gayud ha iya, kakadtuon niya mismo an prinsesa ngan 
Amat, A. B.

said that the Bisayan women wore false hair which they called bangolo or pinanta or tagabohoc, in various regions. The women took great pride in this and in building up a great mass of it and shaping it into a rose below the back-part of the head. Among these women, it is regarded as a great broach of etiquette and serious dishonor to take away this hair or even more, to dishevel it. He mentioned that with it would make a sombol for his ship (we have already mentioned previously that this is a great feathered ornament which they place in the prow as a symbol of victory) and that this would become one of his greatest achievements.

Now, when she learned this news, she fell into tears and began to fear deeply lest he dishonored her (in the above- mentioned fashion). As a result, she decided to prepare the buyos so frequently denied. She had them prepared and placed in a small box of ivory which was exquisitely shaped This was placed inside another small casket, that is, the type that the Principalas have for keeping their jewels. She sent this to him through the black intermediary who so often had come and gone with messages. He told his lord how he had received the buyos, but the master would not receive them or even look at them. Instead, he sent them back stating that he would not accept them entire or whole, but only ground up and those which they call goma and sapa or opa. That, as we have also stated, was a gift of lovers who have won their loved one or the exchange between a wife and a husband, etc. It was then sent in a bujeta of gold and it was a clear sign of their consent and mutual pledge, which we have said was called pugul, for the the desired nuptials. This took place later with pomp and ostentation - depending on their status and wealth.

\section{Cultural Terminologies Used in the Epic}

Agta - is the proper term for the small, black and curly haired people who were the original settler of these islands, in Tagalog term is baluga. In the epic of Alzina agta was the slave of datu.

Apog- in English is lime but in Waray is apog. This is a powder made by burning sea- shells.

Balangay- a native vessel excellent for navigation and transportation.

Bangoloy / Pinanta - more properly Bangoloy and Pinanta from "panta." Both terms mean 'wig' or false hair which the women of that time added to their natural hair thereby building up a beautiful and comely mass.

Binocot-derived from the word Bocot, meaning to "retire or to remain hidden, out of sight." In ancient times, it was usually the wife of a Datu, the Principala who remained so secluded. Among the modern Bisayans the term is now used in reference to monjas or cloistered nuns.

Bonga- is the same as 'fruit' in the Spanish just like "lubi-lubi." The bonga is much smaller than the coconut tree.

$\boldsymbol{B u b u}$ - the author states that the principala or the wife of the Datus was called by this name and that it is of Cebuano origin. In Leyte, the ancient term was seemingly Buro or ovu. paglulugaringan niya pagkurumos an buhok hini. Aton ginhingadayan nga an mga Bisaya nga mga kababayin-an, nakapurungos la ha ira ulo hin diri tuod nga buhok o peke nga buhok nga ginngangaranan nira nga "bangolo" o "pinanta" o diri ngani "tagabohoc" nga matatad-an gihap ini ha magkadurudilain nga dapit. Iginpaparayaw ini han mga kababayin-an, an pagkamay-ada hini, an pagpungos sugad hin bukad hin rosas ha luyo nga ubós la hit ira ulo o tangkugo. Sumala hini nga mga kababayin-an, dako gud kaupay nga kaarawdan kun marabnot ini nga buhok o labi na kun magumok ini. Nagyakan an datu nga pinaagi hito maghihimo hiya hin sombol para hiton iya sakayan. Usa ini nga barahibo hin tamsi na ibinubutang ha unahan hiton sakayan, tigaman hin usa nga kadaugan. Ngan mahihimo nga usa ito han iya pinakadako nga iya nahimo.

Han paghibaro hini han prinsesa, nagnguy-ngoy ini ngan nagtikang an iya kahadlok kay bangin hiya pagpanam tamasan o di ngani pagpalamadyaan. Mahitungod hini, iya dayon igin-andam an mga buyô nga hadto anay nakadamo niya igindiwara Iginbutang niya dida hin gutiay nga surudlan (baul- baol) nga hinimo tikang ha marpil (ivory). Iya liwat ini iginsulod ngadto hin gutiay nga baga hin lungón nga surudlan nga amo an ginhihimusan niya han iya mga alahas. Ug ini iya iginpadara ngadto han datu pinaagi han iya tinapuran nga maitom nga suruguon (agtâ) nga amo pirmi an tagadara han iya mensahe. Iya ginsumatan an iya agaron kun ginpaunanho niya pagkarawat an mga buyô tikang ha prinsesa, kundi waray lugod ito karawata han datu bisan naman la pagsiplat. Lugod iya ini iginpauli ngan nagsiring hiya nga diri niya ini kakarawaton, bug-os man o tanan nga buyô, kundi an pininupino o pinudpod la ngahaw hini nga ira ginngangaranan nga "goma" ngan "sapa" o diri ngani "opa." Ngan aton na ini ginhihingadayan, nga usa ini nga regalo para han hinigugma pagpakita nga nalupig na an iya hinigugma ngan an kabalyo para ha pagkasabot na, o an pagpahimutang na ha pag-asawa. Ipinadará niya ini upod hin bulawan tigaman han han ira paggitangduay nga amo ini an ginngangaranan na "pugol", an pagtigaman han ira ginhihingyap nga kasal. An kasal nahitabo waray pag-iha, makaruruyag ngan duro ka-hurá an pagsalin-urog nga nadepende ini hiton ira maupay nga kabutangan ngan hiton bahandi nga ira naangkon.

\section{Mga Salitang Kultural na Ginamit sa Epiko}

$\boldsymbol{A} \boldsymbol{g t} \hat{\boldsymbol{a}}$ - an iksakto nga tawag han mga gudti-ay, mag-itom, ngan kurong an mga buhok nga mga tawo nga amo an syahan nga nag-ukoy ha mga isla han Pilipinas. Gintatawag gihapon ini hira nga ita o baluga ha Tagalog. Tikang ha susumaton ni Alzina an mga agta in amo an mga kabulig han mga datu.

Apog- ha Englis "lime" pinutos hin buyô o maram-on nga dahon nga may-ada kalugaringon hamot. Usahay tinatawag ini nga "tilad". Pulbos ini tikang ha sinunog nga balayan (shell) hin susô.

Balangay- dinhi-anon nga sarakyan nga maupay para ha pandagat o pambiyahe hadto nga mga kapanahunan.

Bangoloy / Pinanta- tikang ha pulong na "panta".

Karuyag-sidngon, "diri tinuod nga buhok", nga ginagamit han kahadtuan nga mga kababayin-an, basi madugangan an kahusay han pagtukbol han ira buhok.

Binocot- tikang han pulong nga "bocot". Karuyag- sidngon, an diri la anay magpakita, katuyuanan nga kawara o pagtago. Hadto nga panahon, amo ini an tawag han asawa han datu, nga kun diin nag-ukoy ha usa nga mahimyang ngan tago nga lugar. Yana, an tawag hini "monjas."

Bonga - pareho ha Kinatsila nga tawag nga amo an "bonga," sugad han lubi-lubi. An bonga hini in, guruguti kay han lubi.

$\boldsymbol{B u b u}$ - an tagsurat nag-asoy nga an "principala" o an asawa han mga datu, tinatawag ini ha ira ngaran. Kanan Cebuano ini pinulungan. Ha Leyte, an kadaan nga pulong hini in amo an "Buro" o "Ovu". Dinhi hini nga istorya an pulong nga bubu diri ginagamit 
sugad hit nahiasoy dinhi hini nga puplunganon kay it karuyag-sidngon dinhi hit istorya waray pa makaasawa an babaye salit "prinsesa" an mas angay gamiton nga pulong.

Buyo- this is a mixture of one quarter of a bonga (areca) nut, lime (apog) wrapped in a buyo (dapon) or betel leaf which is aromatic. The buyo is also called dalit or tilad in some parts. When the buyo or betel leaf is difficult to acquire, a substitute $L u b a$ serves the same purpose.

Caraga- located on the Eastern coast of Mindanao on a Bay of the same name. This was a region in ancient times known for its very brave, spirited, courageous and strong men.

Camiguin- situated on the north- western coast of the Island of Mindanao; not far from Butuan. It is a rather small island, but it was heavily populated in ancient times. The name is derived from 'cami-guin' or 'our island.'

Ginduutan- derived from Samar - Leyte Bisayan 'Duutan or Dutan,' which means 'to regulate, to adjust, to accord, to compose, to guide, etc. or metaphorically, the horizon.

Goma - this is composed of the three ingredients: the bonga nut, the lime or apog wrapped and tied in the betel leaf or the dapon and chewed to the state of tow until it gives off no more juice whatsoever. The ancient Samareños and Leyteños called it goma while the Spaniards after the Tagalogs, called it sapa. The same Bisayans also had another term for it, upa, which is masticated buyo or that which was begun to be chewed.

Huamianun- a native from Huamian, located in Northern Leyte of Visayas. Just like a native from Palo is called Palon-on, from Tacloban is Taclobanon.

Joangas - this term is derived from the Chinese chun or junco meaning a small vessel used throughout the Asia.

Mangaiao or pangaiao - are derived from the root 'gubat' which means "to fight," to do battle, to engage in hostilities or war." Or again, from the term 'ayao' which means "to complete, or to bring to an end.

Principala - from the Spanish term which means wife of the datus.

Pugul - the word itself means to be afraid of something which seems so overpowering, like a person gone mad. However, metaphorically, it means "to impede, to obstruct, to prevent, to restrain, to hinder or to forbid."

Siquijor or Siquihol - also known as Isla de Fuegos and situated off the Southeast coast of Negros Oriental. It is derived from the words siqui - polo or 'your island.'

Sombol - it is the plumage of a fowl or bird attached to the prow of the ship which forms a curve and which is placed on the vessel which lacks a cutwater, in order to fit the bowsprit to it.

Tagabohoc - the manuscript had 'tagabohoc' which means "just like the hair of the head."

Tandag - situated on the Northeastern coast of Mindanao, in the province of Surigao del Sur.

Yambig - Since no detailed in the map of the region is available it is difficult to ascertain where that islet may be situated. Or perhaps the word is misspelled.
Buy $\hat{\boldsymbol{o}}$ - usa ini nga sinuru-salakot nga bonga (inupat- katipak) ug apog ngan pinutos ini hin kanan buyo dahon nga mahamot. Ha iba nga lugar, an buyô, gintatawag nga "dalit" o "tilad". kun an buyô o dahon makuri bilngon, an "luba" amo an iginsasaliwan hini.

Caraga- nahamumutang ha may Sinirangan nga baybayon han Mindanao. Kinikilala ini nga dapit ha kasanhi dida han mga tuminungnong nga katawhan kumo mga mag-isog, maglaksi, magbangis ug magkusog.

Camiguin- matatad-an ha may Habagat ug Katundan ha isla han Mindanao, diri ini hirayo kaupay ha lugar han Butuan. Haligot la ini nga isla, hadto damo an tawo nga nagpupuyo hini. An ngaran nga Cami-guin, nangangaruyag-sidngon nga "isla namon".

Ginduutan-tikang han Samar- Leyte nga pinulungan - pulong ini han Bisaya nga "Duutan o Dutan" nga an karuyag-sidngon sidsid han langit ngan tuna.

Goma- tulo ini nga magkadurudila-in nga bonga, an apog nga pinutos hin dahon o an dapon nga kun diin ginmamama ini tubtub nga maubos an duga hini. Hadto nga kasanhi, an mga Leyteño ug Samareño ira ini gintawag nga "goma". Samtang an mga Katsila ug mga Tagalog "sapa" an tawag hito. An iba nga mga Bisaya, "upa" an tawag hito nga ginmamama.

Huamianun-an ngaran han tawo nga tuminunong ha Huamian nga matatad-an ha Sinirangan nga Parte han Visayas sugad hit tagaPalo, tinatawag nga Palon-on ngan taga- Tacloban nga Taclobanon.

Joangas- tikang han Inintsek nga pinulungan nga "chun" o "junco", nga an karuyag-sidngon - gutiay nga sakayan - pandagat na amo an gingagamit ha paglayag ha bug-os nga kapurupud-an han Asya.

Mangaiao o pangaiao - tikang ha Englis nga pulong nga "to fight," kun baga ha Waray gumubat," karuyag- sidngon "pakig-away"- pagsulong panuyo hin aragway. Tikang gihap ini ha pulong na "ayao."

Prinsipala- tikang ha Kinatsila nga pinulungan na an karuyag-sidngon, asawa han datu.

Pugul- tikang ha pulong nga "pagpugong" - pagpugol, buotsidngon, kadiri pagtugot ha pagbuhat, pagdaup o pagkadto ha usa nga lugar para pugngan an panuyo. Nangangaruyag-sidngon nga pag-ulang, pagdiri, o kadiri pagtugot.

Siquijor o Siquihol- kilala ini kaupay ha tawag na "Isla de Fuegos". Matatad-an ini ha may dapit han kabaybayunan han Amihan - Sinirangan han Negros Oriental. Tikang ini mga pulong na "siqui" ug "polo" kun baga ha karuyag-sidngon, "imo isla".

Sombol - sugad han pagsayud ni Alzina, ini amo an dagko nga mga barahibo han tamsi, kun ibinubutang ha unahan han sakayanpandagat, naporma ini hin baga pakorbada ug nakakabulig gihap ini pagpalaksi kun tikain an ginsusulsog han sakayan. 


\section{Konklusyon at rekomendasyon}

Batay sa kinalabasan ng pag-aaral na ito, ay nabuo ang sumusunod na konklusyon:

$>$ Sa huling yugto ng basa at suri ng salin ay naisalin at nailapat na ang mga angkop na simulain at prinsipyo ng pagsasaling-wika sa isinaling epiko.

> Nangingibabaw sa epiko ang mataas na paggalang sa kababaihan sa kultura ng panliligaw, ang pagiging matapang at determinadong mangingibig na binata sa dalagang Waraynon, at mataas na pagpapahalaga sa reputasyon at dignidad ng isang Waraynon.

> May mga salitang kultural na nananatili pa rin sa kultura ng mga Waraynon tulad ng buyo, apog, bunga, pangaiao, agta at balangay at may mga salitang nagbago na ang anyo ngunit bahagi pa rin sa kaugalian at pamumuhay ng mga Waraynon katulad na lamang sa pinanta, pugul, balangay. Subalit ang mga salitang binocot, bubu at sombol ay 'di na gamitin sapagkat hindi na ito makikita sa konteksto ng mga Waraynon.

Inerekomenda sa pag-aaral na ito na:

> Gumawa pa ng mga pagsasalin sa Waray sa mga akdang pampanitikang Leynete-Samarnon na nasusulat sa mga wikang Tagalog at Ingles upang ganap itong maintindihan ng mga Waraaynon at magamit na sangguniang babasahin para sa asignaturang panitikang rehiyonal.

> Magsagawa pa ng mga pagsusuri sa mga pagpapahalagang kultural sa mga naisaling akda sa Waray upang mabatid ng mga mag-aaral ang magagandang kaugalian at yamang kultural ng mga Waraynon.

$>\quad$ Magsagawa pa ng etnolinggwistik na pag-aaral sa mga salitang kultural ng Leynete-Samarnon para sa pagpapayaman ng mga salitang lokal at rehiyonal.

\section{Mga sanggunian}

CHED Memo Order No. 20 (2013). https://ched.gov.ph/cmo-20-s-2013/

DepEd Order No. 74 (2009).

https://www.deped.gov.ph/2009/07/14/do-74-s-2009-institutionalizing-mother-tongue-based-multilingu al-education-mle/

Division Memorandum No. 142 (1999). Collection of Waray literature [Kalipunan ng mga Panitikang Waray].

Leyte Normal University. (2008). Rubrics of translation of certificate program for non-specialized in Filipino by the Department of Education [Rubrics ng pagsasalin ng certificate program for non- specialized in Filipino ng Department of Education]. Author.

Ok, H. (2003). Cultural knowledge in translation. ELT Journal, 57(2), 401-429.

Punsalan, T. (1997). Curriculum and instruction: Values education and personality education [Curriculum and instruction: Edukasyon sa pagpapahalaga at edukasyong pagpapakatao]. Values Education Program framework.

Republic Act No. 7356 (1992). https://ncca.gov.ph/republic-act-7356/

Republic Act. No. 10533 (2013). https://www.officialgazette.gov.ph/2013/05/15/republic-act-no-10533/

Tupaz, R. P. (2001). Study activity based on the translation and analysis of selected literature of Region VIII [Gawain ng pag-aaral batay sa pagsasalin at pagsusuri ng mga piling literatura ng Rehiyon VIII] [Unpublished dissertation, Leyte Institute of Technology]. Tacloban City.

Villafuerte, P., Bernales, R. A., \& Protacio, R. M. (2006). Literature of the regions of the Philippines [Literatura ng mga rehiyon sa Pilipinas]. Mutya Publishing House. 